\title{
Design of PM2.5 Detector Circuit Based on Single- Chip Microcomputer
}

\author{
Caiyun Gui ${ }^{1}$ and Baobao Kang ${ }^{2}$
}

\author{
('Yulin University, Yulin $719000^{2}$, BGP INC.,CHINA NATIONAL PETROLEUM CORPORATION, \\ zhuozhou, 071751)
}

Keywords: PM2.5; AT89C52 single-chip microcomputer; LCD12864; Dust sensor

\begin{abstract}
With the advent of the industrial age, the rapid economic development, followed by the deterioration of air quality, the increase in haze and weather and the increase of solid particles in the air seriously affect people's lives. Therefore, it is more and more important to detect solid PM2.5 in the air. The PM2.5 detector is mainly used to detect the particles which are larger than or equal to 2.5 microns in the air, to carry out real-time alarm monitoring and to provide suggestions for people.

This design uses AT89C52 single-chip microcomputer as the main controller, combined with module LCD12864, A/D conversion module, dust sensor module, etc., use dust sensor GP2Y1010AU to convert the current air particulate size into an obvious current or voltage signal, and finally display through the display device which is connected. The design also has alarm function. If the buzzer alarm starts and LED lights on,the dust concentration in the air reaches the set limit. This design circuit is simple with high integration, low power consumption and simple adjustment. The detection accuracy can reach $5 \mathrm{~V} /(\mathrm{mg} / \mathrm{m} 3)$ with high practicality.
\end{abstract}

\section{System overall Plan}

The dust sensor converts the air to be measured into an analog voltage signal and sends it to ADC0832. ADC0832 converts the analog signal obtained into a digital signal and sends it to singlechip microcomputer. The single-chip microcomputer chip sends the input digital signal to LCD and the bee alarm through processing.

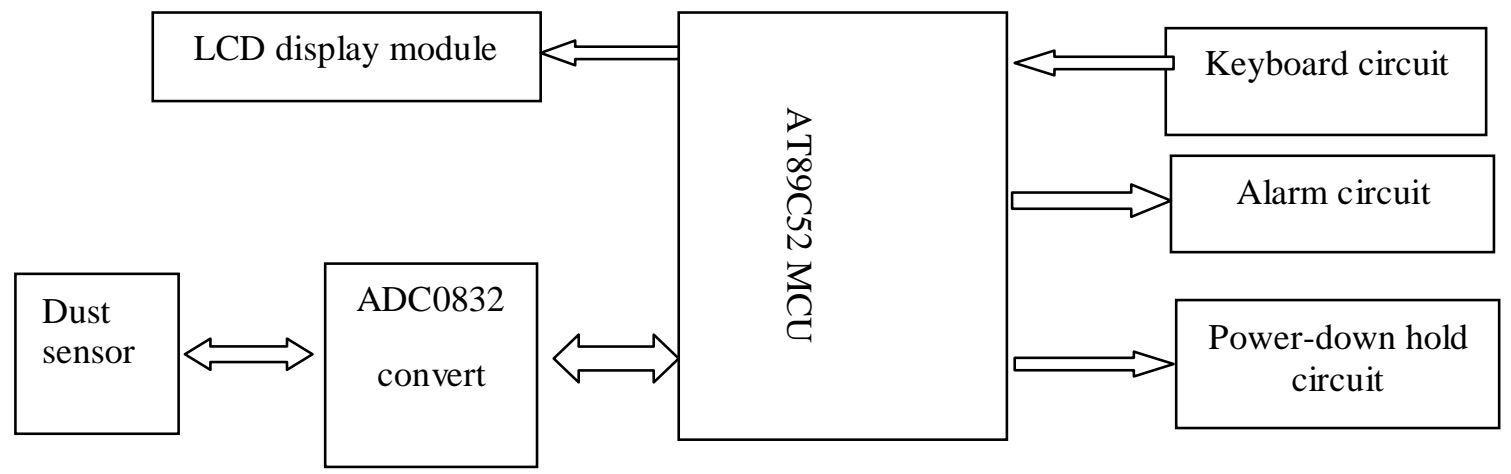

Figure 1. Finite System diagram

\section{PM2.5 Detection Circuit design}

Dust Sensor Circuit Design. The circuit diagram of the dust sensor connected to the microcontroller is shown in Fig. 2. The fifth pin $\mathrm{V}_{0}$ of the device is connected to $\mathrm{AD}$ converter, and the display of the third pin LED is realized through P11 control of single-chip microcomputer. In addition, the second pin and the fourth pin of the sensor are grounded. The first pin of the sensor is realized by a $220 \mathrm{uF}$ capacitor and a resistor ground. The sixth pin of the sensor is connected to the power supply, and the detection of smoke and dust particles is finally implemented. 
Solid suspended particle sensor

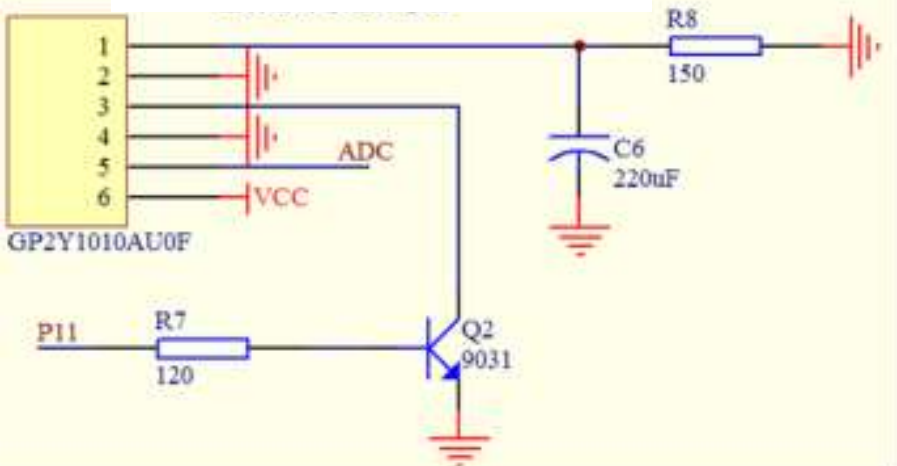

Solid suspended particle sensor

Figure 2. Finite Dust sensor circuit

Data Conversion Module Circuit. After adopting ADC0832 with dual-channel A/D conversion chip, the air pressure sensor converts the air to be measured into analog voltage value, and A/D converter needs to convert the analog voltage value into a digital signal and send it to single-chip AT89C52 for detection and processing and display it on LCD12864. The circuit is shown in Fig. 3.

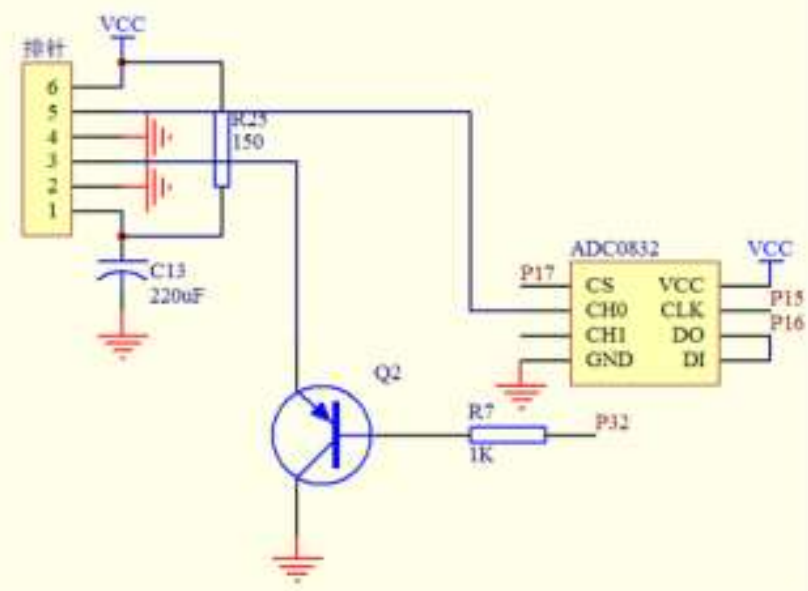

Figure 3. Finite Data conversion module circuit

Display Circuit Design. The dust sensor converts the air to be measured into an analog voltage value, and the analog voltage value is converted into a digital signal by A/D converter ADC0832. This signal is sent to display LCD12864 for processing after being detected and processed by MCU. The circuit diagram of the display module is shown in Fig. 4. Figure P1.0 is connected to the enable terminal of LCD12864; P1.1 is connected to register selection terminal; P2.0 P2.7 are connected to the data line of LCD display, that is the digital signals detected and processed by single-chip AT89C52 is connected to the data line of LCD display through the port of the single-chip microcomputer and sent to LCD display. 


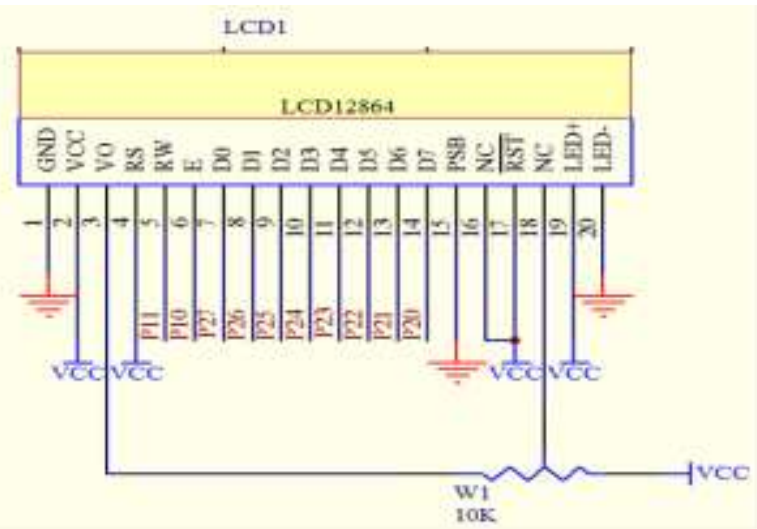

Figure 4. Finite Display circuit

Buzzer Alarm Circuit. Considering the practical value and cost in design, buzzer alarm is used. The schematic diagram is shown in Fig. 5. The buzzer is controlled through I/O port. It should be noted that current limiting resistor connects to buzzer and buzzer cannot be too large, otherwise it is not loud enough. The current limiting resistor is $1 \mathrm{~K}$ ohm.

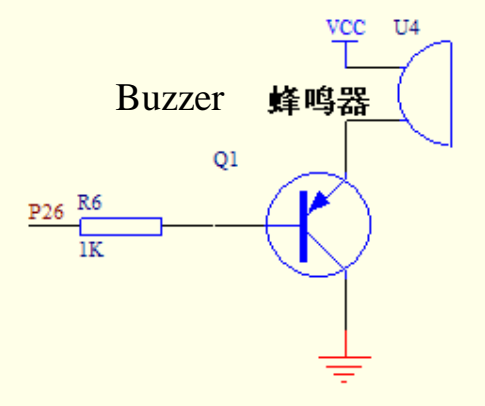

Figure 5. Finite Buzzer alarm circuit

\section{Conclusion}

The design of the hardware circuit includes single-chip minimum system, PM2.5 sensor circuit, data processing circuit, display circuit and buzzer alarm circuit. This paper mainly introduces PM2.5 detection circuit design, including sensor GP2Y1010AU detection circuit, conversion circuit, display and alarm circuit, which can display current air quality in real time and alert people to make travel preparation.

\section{References}

[1] Wu Xuefang, Wang Zongshuang. Suggestions on the Definition of the Names of Airborne Particles in China[J]. Chinese Science and Technology Glossary, 2013, 27(2): 25-28.

[2] Wang Xiaodong. Design of New PM2.5 Detector[J]. Electrical Age, 2014, (4): 82-84.

[3] Liu Aling. Application of Magnetostrictive Displacement Sensor Based on Single-chip Microcomputer[J]. Journal of Ningbo Polytechnic, 2009, (16):19-22.

[4] Zhao Changhao. Research and Design of Successive Approximation ADCs[D]. Chengdu: University of Electronic Science and Technology, 2010:25-38.

[5] Shen Ang. Digital Integration of Space Power Control System[D]. Shanghai: Shanghai Jiaotong University, 2006: 78-80.

[6] Lin Min, Yu Zhongde. Extended LED Display Interface Circuit Based on AT89C2051 Microcontroller Serial Port[J]. Metrology Technology, 2002,(8): 28-29. 